Mots. Les langages du politique

Un sigle politique à haute valeur de marque. Le cas de $Z P$ dans les élections générales en Espagne en 2004

Pablo Medina Aguerrebere et Jordi Rodríguez Virgili

\title{
CpenEdition
}

Journals

Édition électronique

URL : https://journals.openedition.org/mots/20136

DOI : $10.4000 /$ mots. 20136

ISSN : 1960-6001

Éditeur

ENS Éditions

Édition imprimée

Date de publication : 1 mars 2011

Pagination : 99-108

ISBN : 978-2-84788-307-7

ISSN : 0243-6450

Référence électronique

Pablo Medina Aguerrebere et Jordi Rodríguez Virgili, « Un sigle politique à haute valeur de marque. Le cas de ZP dans les élections générales en Espagne en 2004 ", Mots. Les langages du politique [En ligne], 95 | 2011, mis en ligne le 01 mars 2013, consulté le 23 avril 2022. URL : http://

journals.openedition.org/mots/20136; DOI : https://doi.org/10.4000/mots.20136 


\section{Un sigle politique à haute valeur de marque. Le cas de ZP dans les élections générales en Espagne en 2004}

Les candidats et les partis politiques ont besoin d'élaborer un discours leur permettant, d'une part, de transmettre aux citoyens l'essence de leur programme et, d'autre part, de se différencier des autres organisations politiques. Dans ce cadre, plusieurs concepts comme le marketing, l'image de marque, l'identité et le positionnement deviennent, dans une perspective linguistique et sémiotique, des outils pertinents pour l'analyse des campagnes électorales et permettent une approche communicationnelle efficace. La marque des organisations devient un «moteur sémiotique » (Semprini, 1995, p. 47) qui aide à établir avec les consommateurs une «connexion émotionnelle» (Gobé, 2001, p. 306). Ainsi, les actions de communication de marque se basent sur un discours stratégique global qui entraîne de nouvelles manières d'agir et d'interagir, ainsi que de nouvelles formes d'être (Mitsikopoulou, 2008, p. 356-357). Pour analyser cette réalité, notre note de recherche se centrera sur le cas de la campagne électorale conduite en 2004 par l'actuel président du gouvernement espagnol José Luis Rodríguez Zapatero, laquelle s'est fondée sur l'utilisation du sigle ZP (Zapatero Presidente). Nous n'analyserons donc ni la campagne électorale dans son ensemble, ni la stratégie du Parti Socialiste Ouvrier Espagnol (PSOE) et de son candidat, José Luis Rodríguez Zapatero, mais le cas précis de création, d'application et de développement du sigle politique $Z P$. 


\section{Le sigle ZP}

Dans le vocabulaire du marketing, le branding est le processus de construction d'une marque à travers la gestion stratégique de l'ensemble de ses actifs. Dans le cadre d'une campagne électorale, on peut considérer le parti politique et son candidat non seulement comme des marques, mais encore comme des produits (Corner, Pels, 2003; Arvidson, 2006). En Espagne, la campagne ZP Zapatero Presidente de 2004 a constitué un événement dans la publicité politique du pays puisqu'elle a aidé à remplacer la vieille image du PSOE par une image moins formelle, à la fois juvénile et proche de la population. Le célèbre effet $Z P$ et cette campagne électorale novatrice ont réussi à faire d'un candidat presque inconnu un politique d'envergure aux yeux de la population. Ce candidat, en outre, a fini par profiter d'une «marque » ayant un taux de souvenir très important.

Afin d'analyser le cas de ZP en Espagne, on doit replacer dans leur contexte politique les élections générales de 2004. Le leader du Parti Populaire (PP), José María Aznar, quittait la présidence après huit années de gouvernement. Bien qu'il n'existe pas, en Espagne, de limitation au nombre des mandats présidentiels, il avait tenu sa promesse de ne pas se présenter une troisième fois. Le candidat du PP était Mariano Rajoy, le vice-président du gouvernement. Le PP jouissait d'un bon bilan de gestion (associé à la plus importante croissance économique dans l'histoire de la démocratie du pays) et disposait d'un candidat très connu de l'opinion publique. Ses principaux problèmes étaient, d'une part, l'appui à l'intervention militaire en Irak, décision très critiquée par les Espagnols, et d'autre part, la mauvaise gestion de crise du cas Prestige, le pétrolier coulé près des côtes de la Galice en 2002. Malgré tout, les sondages prédisaient la victoire du PP, la seule incertitude portant sur l'ampleur de celle-ci. Ce parti avait donc positionné sa campagne sur le thème de la continuité et dans une stratégie de profil bas.

Le PSOE, lui, avait gouverné de 1982 à 1996. C'est un parti historique profitant d'un fort appui électoral. Pourtant, sa «marque » s'était fortement affaiblie en raison de la corruption et du terrorisme d'État des dernières années du gouvernement de Felipe González. Aux élections générales de l'année 2000, il avait obtenu les plus mauvais résultats de son histoire. En 2004, la principale nouveauté était son candidat, José Luis Rodríguez Zapatero, un homme politique jeune et télégénique. Son tempérament, tel qu'il était perçu, pouvait représenter un nouveau style politique. La priorité de la campagne du PSOE était l'augmentation du taux de reconnaissance de Zapatero.

\section{Concept}

Face à l'usure de la marque du parti et pour rendre Zapatero plus proche de la population, on décida de personnaliser la campagne en mettant l'accent sur 
le candidat. L'approche personnalisante, basée sur des stratégies expérimentées aux États-Unis, représente un pas de plus vers la professionalisation des campagnes électorales. Les candidats deviennent des produits, des vedettes du spectacle. En 1952, dans la campagne d'Eisenhower contre Stevenson, considérée comme pionnière dans la perspective qui nous occupe, les républicains ont conçu - grâce à sa simplicité, sa musicalité et sa fraîcheur - l'un des meilleurs slogans de l'histoire du marketing politique, I like Ike. Cette campagne a constitué le commencement du branding appliqué à un candidat. En Espagne, ce genre de branding a vu le jour en 2004 avec la création du sigle ZP. On a élaboré une identité institutionnelle différente de celle du PSOE, afin de développer une campagne basée sur l'image de jeunesse, de proximité et de modernité que projetait José Luis Rodríguez Zapatero. On confrontait avantageusement celle-ci à l'image de José María Aznar, très érodée après un dernier mandat marqué par le coût politique de la guerre en Irak, et à celle de Mariano Rajoy, un politique gris, très connu mais dont le profil professionnel ne représentait que la continuité.

La marque du parti politique, qui a pour objectif de positionner le parti et ses valeurs dans l'esprit des électeurs, ne s'élabore pas au cours d'une campagne électorale : elle se construit progressivement et sur le long terme, tout comme les marques commerciales. Les campagnes s'appuient sur la marque du parti, mais se centrent sur les messages et sur le candidat. C'est ainsi que l'adaptation des techniques commerciales à la communication politique a donné lieu au style américain, c'est-à-dire à l'utilisation de la musique, des spots publicitaires, de la théâtralisation et de la médiatisation des débats entre les candidats, etc. (Berrocal, 2003; Maarek, 2009). La professionnalisation des campagnes a renforcé le rôle des leaders ou l'usage des sound bites (Holtz-Bacha et al., 2007).

La personnalisation de la marque politique accélère les facteurs qui déterminent le succès des campagnes. Aux États-Unis, sur 506 spots diffusés entre 1960 et $1984,15 \%$ seulement transmettaient des informations sur les propositions politiques, tandis que $57 \%$ étaient centrés sur les qualités personnelles et professionnelles des candidats comme, par exemple, son image (McNair, 2003, p.90). La personnalisation est un élément propre du système présidentialiste américain, alors qu'en Espagne, l'action la plus caractéristique est la mise en avant du programme du parti politique. Pourtant, la tendance à la personnalisation des spots s'implante progressivement dans ce pays aussi, et plusieurs études confirment la préférence des électeurs pour les aspects liés à la personnalité des candidats au-delà de l'idéologie (López-Escobar et al., 1996). Dans ce cadre, la décision du PSOE de personnaliser sa campagne constituait, d'une part, une continuité logique et, d'autre part, une nouveauté un peu hasardeuse.

La campagne a été gérée par l'agence Think Small, créée exprofesso pour ce projet. Cette agence, dirigée par Juan Campmany, a trouvé un slogan concret, simple, compréhensible et impliquant le récepteur: Nous méritons une Espagne meilleure (Merecemos una España mejor). On acceptait du bout des lèvres la 
bonne situation du pays, mais on rêvait de quelque chose de mieux après les erreurs du gouvernement concernant l'Irak et la catastrophe du Prestige. II fallait ensuite mettre en avant le candidat, dont le prénom et le nom de famille étaient très longs et très courants, ce qui exerçait une contrainte sur la créativité. L'agence décida alors de parier sur son second patronyme : Zapatero.

L'équipe de publicitaires découvrit par ailleurs un élément que seul le PSOE pouvait développer d'une manière stratégique. La campagne du PP était très marquée par l'existence parallèle de deux points d'intérêt : le président Aznar et le candidat Mariano Rajoy. Le PP présentait Rajoy comme le candidat à la présidence du gouvernement, laquelle était encore occupée par le président Aznar. De son côté, Zapatero représentait à lui seul son parti politique, d'où l'utilisation de Zapatero Presidente. Il s'agit là d'un recours très habituel dans les campagnes américaines (Clinton for President), déjà utilisé en Espagne par Miquel Roca (Convergència Democràtica de Catalunya) dans les élections de 1979 (Roca for President). Le message n'est pas passé inaperçu, il transmettait une sensation de sécurité et de confiance dans le changement, il était perçu comme étant un peu provocateur (selon les sondages, le PSOE était derrière le $\mathrm{PP}$ ) et il exigeait une mise en scène frappante.

On avait conçu le slogan central de la campagne, mais il fallait encore créer un concept graphique et audiovisuel. L'équipe de publicitaires trouva l'idée du sigle ZP lors d'une session de brainstorming consacrée à la stratégie de présentation du slogan. Tandis qu'elle réfléchissait au concept à utiliser, le directeur artistique, Marc Conca, se mit à gribouiller les esquisses d'un panneau publicitaire exhibant le profil de José Luis Rodríguez Zapatero et le slogan central en minuscules. Il respectait par là le choix que l'agence avait fait de présenter les textes de façon claire, en les centrant sur la recherche d'un face-à-face avec le récepteur. Ce choix conduisait à éviter, si possible, l'utilisation d'une capitale au premier caractère, ce qui aurait dénoté une image trop formelle. Mais, le directeur artistique respectait la majuscule à l'initiale dans le slogan :

À un certain moment de cette longue session, après 22 heures et à l'instant où tout le monde souhaitait, au moins, faire une pause pour le dîner, j’ai réalisé l'importance de ce détail-là. À ce moment, j’ai remarqué qu'il y avait deux caractères, le $Z$ et le $P$, qui ressortaient de l'ensemble. À l'instant, quelqu'un a ajouté, en se parlant à soi-même mais en le faisant à haute voix : Zetapé. Et un troisième membre de l'équipe a fait une référence au mythique JFK du président Kennedy. Il s'agissait d'un échange d'impressions et d'idées qui a duré moins d'une minute, mais qui a servi à déclencher l'enthousiasme, même si on n'était pas encore conscients de cette trouvaille. (Campmany, 2005, p. 34)

J'ai demandé à Marc Conca de dessiner un modèle portant les sigles $Z P$ au-dessus de Zapatero Presidente. À partir de ce moment, les esquisses de Marc se sont multipliées, toutes accordant une place importante à $Z P$. On a inséré ce sigle dans des formats verticaux et horizontaux, dans des affiches avec et sans photographies. On a essayé un modèle portant uniquement $Z P$, «comme s'il s'agissait d'un badge 
ou d'un autocollant... », a souligné l'un des participants. Dans une ambiance où l'adrénaline nous avait fait oublier la fatigue et la faim, on a conclu, presque sans le besoin de le dire d'une manière précise, qu'on avait déniché le meilleur format pour notre slogan de campagne. (Ibid.)

C'est ainsi que fut créé un logo original et sonore, un emblème, une marque personnelle cohérente avec le biais présidentialiste que le PSOE souhaitait imprimer à la campagne. Le sigle ZP, Zapatero Presidente, synthétisait la fierté avec laquelle ce parti présentait son candidat. Ses créateurs avaient l'ambition de motiver l'électorat en lui instillant la conviction que la victoire était possible, malgré les sondages. Le sigle $Z P$ entrait en contact, notamment, avec les jeunes parce qu'il était cohérent avec la tendance communicative basée sur la création de sigles dynamiques et proches de leur langage. Cette tendance avait été promue par des émissions de télévision, véritables phénomènes médiatiques juvéniles et leaders d'audience comme OT (Operación Triunfo) et GH (Gran Hermano). Le sigle était en outre cohérent avec le langage qu'utilisent les jeunes dans les messages envoyés par téléphone portable, lesquels sont très marqués par l'usage de sigles, de pictogrammes et de mots sans voyelles.

\section{Diffusion}

Le 12 février, les socialistes lancèrent la campagne, avec ses différents contenus et supports, auprès de l'opinion publique. Elle visait à confronter la modernité et le dynamisme de Zapatero à « la vieillesse et l'immobilisme » représentés par Rajoy (El País, 13 février 2004). Dans cette idée, on conçut des initiales de très grandes dimensions, et dans la plupart des meetings politiques tenus à cette occasion, de larges panneaux frappés du sigle $Z P$ attiraient l'attention des caméras de télévision et des photographes de presse (voir image ci-dessous). C'était la meilleure manière de visualiser l'étoffe présidentielle du candidat: le PSOE parvint ainsi à produire une campagne « directe, mobilisatrice et positive» (El Mundo, 13 février 2004).

Les créateurs souhaitaient que ZP, Zapatero Presidente exprime la continuité, l'unité et la lisibilité de deux concepts, l'un réel (Zapatero) et l'autre potentiel (Presidente). C'était un sigle frappant, qui représentait les formes consonantes d'un nom ainsi qu'un objectif. Les attributs de ce sigle se centraient sur le tempérament du candidat et sa capacité au dialogue. La marque répondait à une stratégie d'identité, non pas unitaire ou monolithique, mais diversifiée. Le PSOE a donc créé une stratégie distribuée, très similaire à celle d'une entreprise qui dispose de plusieurs produits, et dessiné une marque spécifique pour le sien (Zapatero). On a respecté les normes de la marque mère (PSOE), mais on a aussi présenté une identité nouvelle pour un produit nouveau qui, quelque part, se différenciait du produit précédent (c'est-à-dire des candidats socialistes antérieurs). Et l'axe graphique constituait la manière la plus simple de communiquer ce changement. 
Le sigle ZP fut mis en avant dans les applications de publicité extérieure (banderoles, affiches pour les cabines téléphoniques, etc.) et les matériaux destinés à la diffusion massive (les autocollants, par exemple). Afin de souligner son importance, chacune des initiales du sigle et du mot qui la développait (Zapatero ou Presidente) était d'une couleur différente : le rouge et le noir, respectivement, sur fond blanc; ou le blanc et le noir sur fond rouge ${ }^{1}$. L'équipe de publicitaires utilisa ces deux fonds en alternance. Tantôt on respectait le fond blanc habituel, expression de la transparence et de la netteté du message et de ses émetteurs. Tantôt on utilisait le fond rouge institutionnel du PSOE, avec le dynamisme et le courage symbolisés par cette couleur, qu'utilisent d'autres produits ayant un taux de vente important (Coca-Cola, Marlboro, etc.). On joua sur l'opposition avec les caractères en blanc du sigle pour compenser les connotations d'agressivité du rouge. Le sigle fut en outre adapté à différents formats, comme le montre l'usage d'une ou de deux lignes pour les mots Zapatero Presidente, afin de changer la forme rectangulaire du logo par une forme quadrangulaire.

Dans la publicité, on a choisi pour le rouge du $Z$ et du mot Zapatero le pantone pur (\#FFoooo), quoique le PSOE utilise couramment le pantone rouge \#EF1920 (ou rouge socialiste), notamment pour son logo. Le noir, en revanche, est proche du gris foncé (pantone ${ }_{44} \mathrm{~A}_{4} \mathrm{~A} 4 \mathrm{~A}$ ). La typographie appartient a la famille des arial (la sans-serif plus standard), modifiée dans les mots Zapatero Presidente : pour Zapatero et la lettre $Z$ du sigle, on s'est servi d'une arial bold, d'après le logo du PSOE, qui utilise le bold dans les sigles du parti. Mais on a négativisé l'effet (rouge sur blanc au lieu de blanc sur rouge au PSOE) et on s'est passé du rétrécissement (narrow) des lettres du logo de ce parti².

On a par ailleurs créé un site internet consacré à la campagne électorale (http://www.zapateropresidente.com, aujourd'hui obsolète). Afin de faciliter sa consultation par les usagers, on a lié la recherche des caractères $Z P$ dans Google à l'ouverture dudit site. Nous proposons en annexe à cette note deux photographies diffusées par le département de communication du PSOE.

\section{Résultats obtenus}

Dès le lancement de la campagne, le sigle $Z P$ a attiré l'attention de la presse sur le phénomène du marketing électoral. Aux yeux des médias, la campagne paraissait d'une grande nouveauté. Les Espagnols, eux, ne prirent pas tout de suite le sigle au sérieux et en firent l'élément principal de plaisanteries ou de calembours : shampooing ZP, Zozobra-Política ("Naufrage-Politique »), etc. Mais c'était là une preuve claire de l'impact du message. Le PP, lui aussi, pro-

1. Voir photo en annexe.

2. Voir logo en annexe. 
duisit d'abord des blagues sur ce sigle, ce qui contribua à sa diffusion; trois jours après, il comprit la situation et mit fin à ce genre d'actions, mais il était trop tard. La blague était un indice de la reconnaissance et de la notoriété du sigle. Le slogan, bref et mémorisable, avait atteint ses objectifs : visibilité, notoriété, opportunité de présenter un candidat plus populaire et plus proche des électeurs. La proximité était la clé de la stratégie : selon Christian Le Bart (2005, p. 18), elle «permet de puiser dans un univers de connotations positives qui présente l'avantage de ne pas risquer de déplaire».

Les jeux de mots créés par les citoyens proches de la droite politique espagnole continuèrent à fleurir pendant la campagne électorale : ZapateroPerdedor («Zapatero-Perdant»), Zapatero-Paleto («Zapatero-Ignorant»), Zapatero-Patético («Zapatero-Pénible), etc. Ces railleries aidèrent à la diffusion du concept et même à la neutralisation de ce genre de blagues. Invité d'un talkshow très réputé en Espagne, El programa de Fuentes, où il adopta le ton de Bill Clinton afin d'atteindre les électeurs les moins politisés, José Luis Rodríguez Zapatero fit des allusions au concept $Z P$ à maintes reprises. Il alla jusqu'à révéler qu'étant écolier, il avait dû supporter les blagues que ses camarades faisaient sur son nom de famille. Il profita de cette occasion pour contre-attaquer les railleries du PP. Quant aux adversaires qui identifiaient la marque $Z P$ avec celle d'un shampooing ou d'un insecticide, il leur répondit que $Z P$ était un produit destiné à éliminer les pellicules semées par le PP.

L'image de leadership de Zapatero au sein du PSOE, diffusée et popularisée par le sigle $Z P$ pendant la campagne électorale, et la comparaison avec le débat interne existant au sein du PP entre Aznar et le candidat Rajoy ont pu déterminer trois conséquences : centrer l'intérêt de la campagne sur le candidat socialiste, ce qui augmenta sa visibilité; renforcer son image de leader face au leadership usé des candidats socialistes depuis la défaite électorale de 1996; et, finalement, capitaliser les voix anti-Aznar.

\section{Évolution}

Le sigle $Z P$ continua d'être utilisé après la campagne électorale, mais le pari risqué qui avait si bien marché lors des élections de 2004 devint une idée frivole qui ne fut plus utilisée lors des élections générales de 2008. Cette année-là, le PSOE a fait évoluer le sigle et créé le slogan Avec le $Z$ de Zapatero (Con Z de Zapatero). Zapatero jouissait alors d'une meilleure image que son adversaire, à nouveau Mariano Rajoy, et c'est pourquoi les responsables de campagne du PSOE mirent en place une stratégie axée sur le choix entre les deux leaders. La population espagnole continua d'associer le sigle $Z P$ avec le patronyme Zapatero et lui seul, plutôt qu'avec Presidente. Les marques de produit doivent être consistantes et persévérantes lors du processus de construction de leur personnalité. Cela n'a pas été le cas de la marque $Z P$ : quelques mois après les 
élections de 2004, les adversaires du leader socialiste étaient les seuls à utiliser le sigle pour nommer le président du gouvernement.

Aujourd'hui, plus de deux ans après le début du deuxième mandat de José Luis Rodríguez Zapatero, le sigle ZP n'a plus de valeur ajoutée et, d'ailleurs, nuit à la marque du PSOE, laquelle, par contre, jouit d'une meilleure réputation. Zapatero a écrit une page dans l'histoire politique espagnole puisqu'il a été le seul président à obtenir les meilleurs et les pires évaluations. Depuis février 2009, il est le président ayant le pire taux de confiance de toute l'histoire démocratique espagnole. Cette réalité peut entraîner un vrai problème pour le PSOE, à cause de la stratégie de personnalisation qu'il a choisie.

L'utilisation de sigles en politique peut apporter une valeur décisive à un parti ainsi qu'à son candidat, parce qu'ils mettent en avant la valeur sémiotique de la marque. Cela non seulement détermine le discours global du parti politique, mais encore apporte une composante de personnalisation et de créativité qui permet de renforcer un positionnement stratégique. Dans le cas de $Z P$, trois dernières idées permettent de conclure. En premier lieu, l'utilisation de ce sigle constitue un pas vers la professionalisation du marketing politique espagnol, en même temps qu'une nouveauté intéressante puisque c'est la première fois qu'un parti construit une telle marque pour son candidat. En deuxième lieu, il est difficile d'évaluer les effets politiques de cette campagne à cause du rôle qu'y ont joué les attentats terroristes du 11 mars 2004; mais, d'un point de vue communicatif, elle a été un succès puisqu'elle a atteint ses objectifs principaux : visibilité de José Luis Rodríguez Zapatero et transmission d'une image conforme à certaines attentes. En troisième lieu, la campagne $Z P$ ne constitue pas pleinement une création de marque, et en reste plutôt au stade du slogan, puisqu'elle ne respecte pas la condition de succès la plus importante dans le monde des marques : la suite communicative.

\section{Références}

ArVIDson Adam, 2006, Brands, Meaning and Value in Media Culture, Londres, Routledge. Berrocal Salomé, 2003, Comunicación política en televisión y nuevos medios, Barcelone, Ariel.

CAMPMANy Juan, 2005, El efecto Zp. 1000 días de campaña para llegar a la Moncloa, Barcelone, Planeta.

CORner John, Pels Dick éd, 2003, Media and the Restyling of Politics. Consumerism, Celebrity and Cynicism, Londres, Sage.

El Mundo, 13 février 2004, «El PSOE dice que si se celebraran debates podría suprimir el $50 \%$ de la campaña y dar el dinero a ONGs», en ligne [http://www.elmundo. es/elmundo/2004/02/12/espana/1076590042.html], consulté le 18 janvier 2010. El País, 13 février 2004, «Zapatero promete la vuelta de las tropas el 1 de julio si la ONU no se 
hace cargo de Irak», en ligne [http://www.elpais.com/articulo/espana/Zapatero/promete/vuelta/tropas/julio/ONU/hace/cargo/Irak/elpepiesp/20040213elpepinac_6/ Tes/], consulté le 15 janvier 2010.

Gobé Marc, 2001, Emotional Branding. The New Paradigm for Connecting Brands to People, New York, Alworth Press.

Holtz-Bacha Christina, Negrine Ralph, Mancini Paolo, Papathanassopoulos Stylianos éd., 2007, The Professionalisation of Political Communication, Chicago, Intellect Books.

LE BART Christian, 2005, "La proximité selon Raffarin», Mots. Les langages du politique, $\mathrm{n}^{0} 77, \mathrm{p} .13-28$.

López-Escobar Esteban, McCombs Maxwell, Rey Federico, 1996, «La imagen de los candidatos. El segundo nivel de la agenda-setting», Comunicación y Sociedad, $\mathrm{n}^{\circ} 9$, p. 39-65.

MAArek Philippe J., 2007, Communication et marketing de l'homme politique, Paris, LexisNexis (Marketing político y comunicación. Claves para una buena información política, trad. P. Multinger, Barcelone, Paidós, 2009).

Mitsıkopoulou Bessie, 2008, «The branding of political entities as discursive practice », Journal of Language and Politics, vol. III, nº 7, p. 353-371.

MCNaIR Brian, 2003, An Introduction to Political Communication, Londres, Routledge.

SEMPRINI Andrea, 1992, Le marketing de la marque. Approche sémiotique, Paris, Liaisons (El marketing de la marca. Una aproximación semiótica, trad. T. Goñi, Barcelone, Paidós, 1995). 


\section{Annexe}

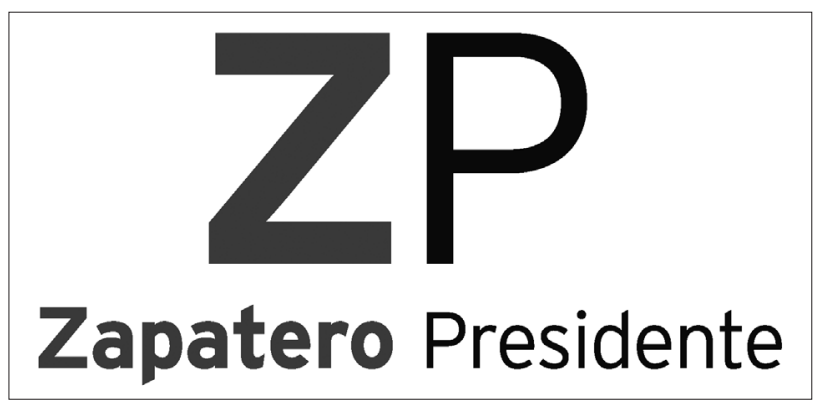

Le sigle ZP de la campagne.

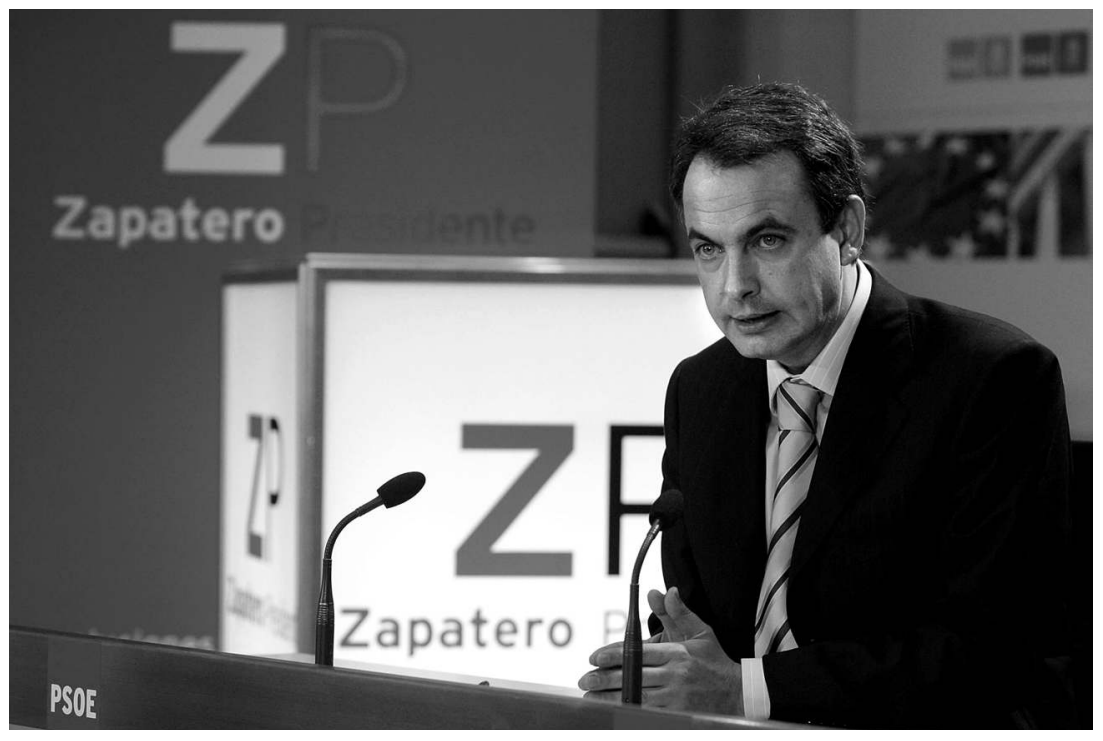

La mise en scène des «sceaux » et du sigle $Z P$ dans un meeting de José Luis Rodríguez Zapatero. 